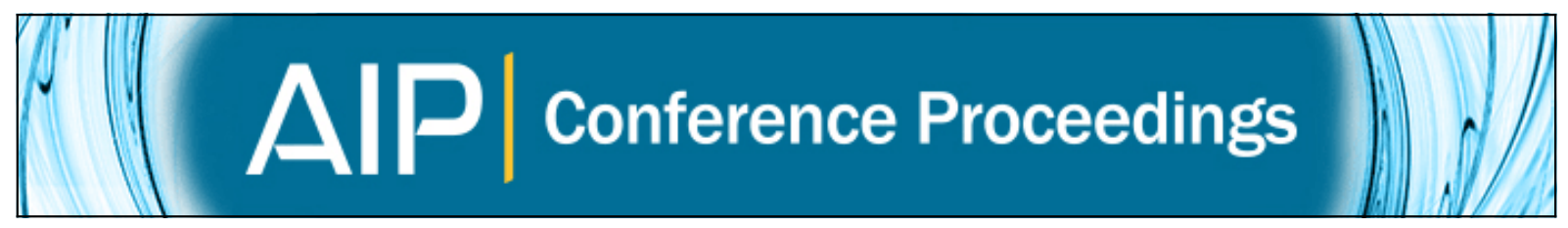

A double inversion: Size and time resolved growth rates for aerosol particles in the CERN CLOUD experiment

C. Williamson, J. Curtius, A. Kürten, J. Almeida, S. Ehrhart, D. Wimmer, K. Lehtipalo, J. Tröstl, and CLOUD Collaboration

Citation: AIP Conference Proceedings 1527, 389 (2013); doi: 10.1063/1.4803285

View online: http://dx.doi.org/10.1063/1.4803285

View Table of Contents: http://scitation.aip.org/content/aip/proceeding/aipcp/1527?ver=pdfcov

Published by the AIP Publishing

Articles you may be interested in

Thermophoresis of aerosol particles in near-critical vapor: An inverse size effect

Appl. Phys. Lett. 106, 251605 (2015); 10.1063/1.4923198

Aerosol nucleation and growth in a mixture of sulfuric acid/alpha-pinene oxidation products at the CERN CLOUD chamber

AIP Conf. Proc. 1527, 322 (2013); 10.1063/1.4803268

Atmospheric nucleation and growth in the CLOUD experiment at CERN

AIP Conf. Proc. 1527, 278 (2013); 10.1063/1.4803258

Growth of Charged Particles in Clouds

J. Appl. Phys. 19, 1053 (1948); 10.1063/1.1698009

CERN's CLOUD experiment

Phys. Today 


\title{
A Double Inversion: Size and Time Resolved Growth Rates for Aerosol Particles in the CERN CLOUD Experiment
}

\author{
C. Williamson ${ }^{\mathrm{a}}$, J. Curtius ${ }^{\mathrm{a}}$, A. Kürten ${ }^{\mathrm{a}}$, J. Almeida ${ }^{\mathrm{a}}$, S. Ehrhart ${ }^{\mathrm{a}}$, \\ D. Wimmer ${ }^{\mathrm{a}, \mathrm{b}}$, K. Lehtipalo ${ }^{\mathrm{b}}, \mathrm{J}^{\text {. Tröstl }}{ }^{\mathrm{c}}$ and the CLOUD collaboration. \\ ${ }^{a}$ Insitut für Atmosphäre und Umwelt, Goethe University of Frankfurt, Frankfurt am Main, Germany \\ ${ }^{b}$ Department of Physics, University of Helsinki, Helsinki, Finland \\ ${ }^{c}$ Paul Scherrer Institut, Laboratory of Atmospheric Chemistry, Villigen, Switzerland
}

\begin{abstract}
An integrated analysis of multiple instruments measuring concentrations of aerosol particles in the CLOUD (Cosmics Leaving OUtdoor Droplets) chamber at CERN obtains size and time dependent growth rates. A matrix inversion is performed twice: first to get size distributions from measured concentrations using instrument transfer functions; secondly to get growth rates from size distributions using the aerosol general dynamic equation (GDE).
\end{abstract}

Keywords: Aerosols, Growth Rates, Chamber Experiments, Inversion Methods

PACS: $92.60 . \mathrm{Mt}$

\section{THE CLOUD-EXPERIMENT}

Ion-induced nucleation is one mechanism proposed to explain a possible link between galactic cosmic rays (GCRs) and cloud formation ${ }^{[1]}$. Along with the role of ternary species, this can be better understood through analysis of aerosol particle growth rates under controlled conditions. CLOUD aims to perform this analysis using an aerosol chamber containing synthetic air, controlled levels of sulfuric acid, amines and organics ${ }^{[2]}$.

Data was collected over a two month measurement campaign in 2012. Aerosol nucleation and growth rates were investigated under various conditions including: ternary nucleation with ammonia/DMA, pure ozonolysis and pure $\mathrm{OH}$ oxidation of $\alpha$ pinene and simulation of atmospheric conditions at SMEAR II ${ }^{[3]}$. Neutral, GCR and CERN-PS beam conditions were used to study ion effects. Many instruments continuously measured particle concentrations in the chamber, including: two 
diethelene-glycol condensation particle counters (DEG-CPCs) ${ }^{[4]}$, a scanning and a fixed particle-size-magnifier (PSM) ${ }^{[5]}$, a CPC-battery (TSI CPCs 3776, 3772, 3010), a laminar diffusion tube (LDT) ${ }^{[6]}$ and a scanning mobility particle sizer (SMPS) ${ }^{[7]}$.

\section{THE INVERSION}

\section{Inversion 1: from Concentrations to Size Distributions}

Each instrument or instrument channel measures particles of varying sizes with varying counting efficiencies, characterized by transfer functions as shown in figure 1. These transfer functions, $f_{i}\left(D_{p}\right)$, relate the measured concentrations, $\mathrm{b}$, to the size distribution, $\frac{d N\left(D_{p}\right)}{d \log D_{p}}$, as follows:

$$
f_{i}\left(D_{p}\right) \frac{d N\left(D_{p}\right)}{d \log D_{p}} d \log D_{p}=b_{i}
$$

Eq.1 is discretized giving a matrix, A, of the transfer functions, whose pseudoinverse is found using singular value decomposition. Multiplying this with the vector of measured concentrations, $\vec{b}$, gives a set of possible size distributions, $\vec{x}$ :

$$
\vec{x}=A^{-1} \vec{b}+V \vec{a}
$$

where $\boldsymbol{V}$ is the null-space of $\boldsymbol{A}$ and $\vec{a}$ is an arbitrary vector.

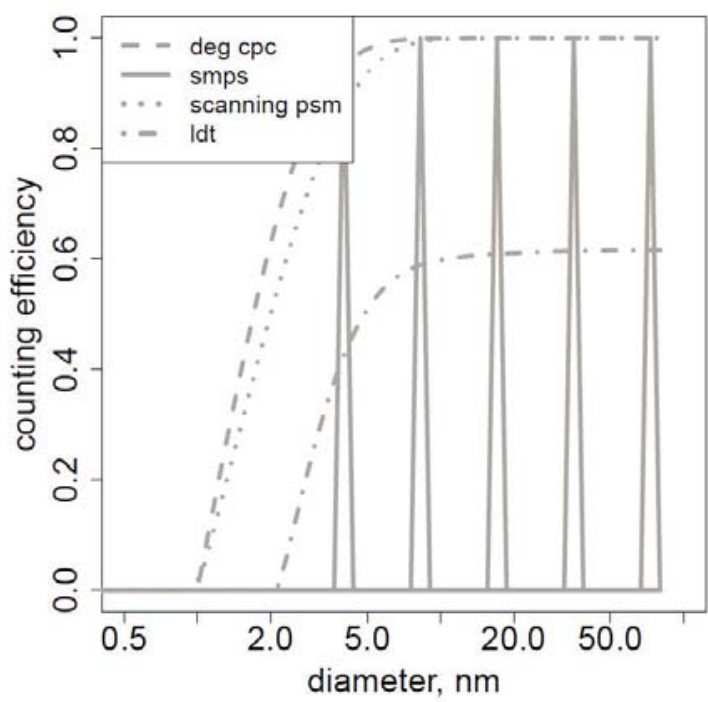

FIGURE 1. Transfer functions from instruments measuring particle concentrations in the CLOUD chamber. Only selected channels are shown, namely DEG CPC 1, SMPS channels $4.0,8.3,17.1,35.5,55.0 \mathrm{~nm}$, scanning PSM 0.16lpm flow, LDT 1550cm3/min flow. 
A solution is found assuming nothing about the functional form of the solution except that the size distribution is non-negative and smooth, correcting for inherent bias to larger size bins and weighted each measurement according to its uncertainty ${ }^{[8]}$.

Before application to data from CLOUD, this method was tested on simulated size distributions. Expected instrument responses for each size distribution were calculated using transfer functions, varied within instrumental uncertainty and run through the inversion method. The input and inverted size distributions can then be compared as shown in figure 2 .

\section{Inversion 2: from Size Distributions to Growth Rates}

The GDE for aerosol particles can be expressed as follows ${ }^{[9]}$ :

$$
\frac{d N_{\Delta}(t)}{d t}=\frac{d N_{\Delta}}{d D_{p}}\left(D_{p 1}, t\right) \frac{d D_{p 1}}{d t}-\frac{d N_{\Delta}}{d D_{p}}\left(D_{p 2}, t\right) \frac{d D_{p 2}}{d t}+C_{\Delta}\left(D_{p 1}, D_{p 2}, t\right) \quad \text { Eq. } 3
$$

Where $\Delta$ is the size interval between particle diameters $D_{p 1}$ and $D_{p 2}$ and $\frac{d D_{p}}{d t}$ is the growth rate. The combined coagulation sources and sinks for each interval $\left(C_{\Delta}\right)$ are calculated using the Fuchs coagulation rate coefficient ${ }^{[10]}$.

Eq. 3 can be discretized and rearranged to take on the following vector form

$$
\frac{d N_{\Delta}}{d D_{p}} \overrightarrow{G R}=\frac{\overrightarrow{d N_{\Delta}(t)}}{d t}-C_{\Delta}
$$

This is inverted in the same way as eq. 2 to retrieve the vector of growth rates, $\overrightarrow{G R}$.

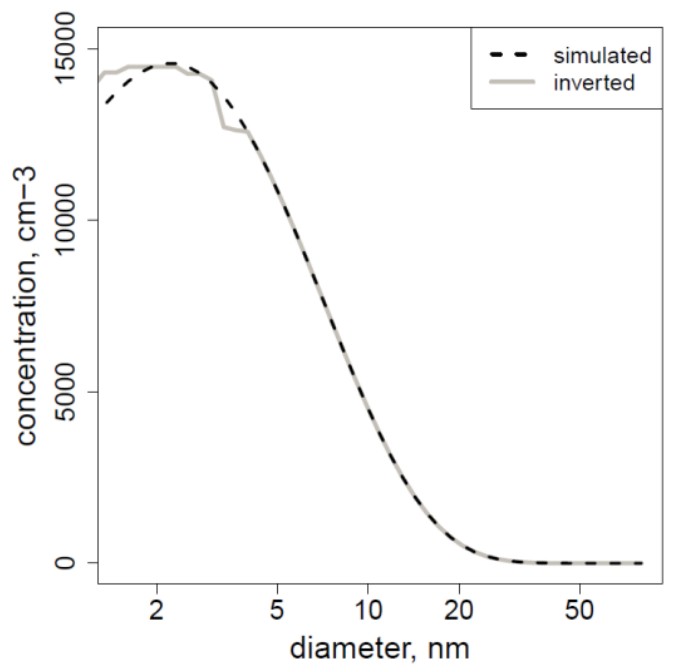

FIGURE 2. A simulated size distribution (dashed black) and an inversion of predicted concentrations obtained from this distribution (solid grey). 


\section{ACKNOWLEDGMENTS}

We would like to thank CERN for supporting CLOUD with important technical and financial resources, and for providing a particle beam from the CERN Proton Synchrotron. This research has received funding from the EC Seventh Framework Programme (Marie Curie Initial Training Network "CLOUD-ITN" no. 215072, MCITN "CLOUD-TRAIN" no. 316662, and ERC-Advanced "ATMNUCLE" grant no. 227463), the German Federal Ministry of Education and Research (project nos. 01LK0902A and 01LK1222A), the Swiss National Science Foundation (project nos. 200020_135307 and 206620_130527), the Academy of Finland Center of Excellence program (project no. 1118615), the Academy of Finland (CoE project no. 1118615, LASTU project no. 135054), the Nessling Foundation, the Austrian Science Fund (FWF; project no. P19546 and L593), the Portuguese Foundation for Science and Technology (project no. CERN/FP/116387/2010), the Swedish Research Council, Vetenskapsrådet (grant 2011-5120), the Presidium of the Russian Academy of Sciences and Russian Foundation for Basic Research (grants 08-02-91006-CERN and 12-02-91522-CERN), and the U.S. National Science Foundation (grants AGS1136479 and CHE1012293).

\section{REFERENCES}

1. K. Carslaw et al. Cosmic Rays, Clouds, and Climate, Science, 298, 1732-1737 (2002).

2. J. Kirkby et al. Role of Sulphuric Acid, Ammonia and Galactic Cosmic Rays in Atmospheric Aerosol Nucleation, Nature, 476, 429-433 (2011).

3. P. Hari et al., Station for Measuring Ecosystem-Atmosphere Relations (SMEAR II), Boreal Environ. Res., 10, 315-322 (2005).

4. D. Wimmer et al, Performance of Diethylene Glycol Based Particle Counters in the Sub 3nm Size Range, submitted to Atmos. Meas. Tech. Discuss January 2013.

5. J. Vanhanen et al. Particle Size Magnifier for Nano-CN Detection, Aerosol Sci. Technol., 45:4, 533-542 (2011).

6. C. Tan and C Hsu, Diffusion of Aerosols in Laminar Flow in a Cylindrical Tube, Aerosol Sci., 2, 117-124 (1971).

7. S. Wang and D Flagan, Scanning Electrical Mobility Spectrometer, Aerosol Sci. Technol., 13, 230240 (1990).

8. M. Fiebig et al. Inversion of Data Containing Information in the Aerosol Particle Size Distribution Using Multiple Instruments, Journal of Aerosol Sci., 36, 1353-1372 (2005).

9. C. Kuang, et al. Size and Time-Resolved Growth Rate Measurements of 1 to $5 \mathrm{~nm}$ Freshly Formed Atmospheric Nuclei, Atmospheric Chemistry \& Physics, 3573-3589 (2012).

10. N. Fuchs, The Mechanics of Aerosols, Pergamon Press, Oxford (1964). 